\title{
Aeroacoustic Investigation of Trailing-Edge Finlets
}

\author{
Yehia Salama, Joana Rocha \\ Department of Mechanical and Aerospace Engineering, Carleton University, Ottawa, Canada \\ Email: YehiaSalama@cmail.carleton.ca, Joana.Rocha@carleton.ca
}

How to cite this paper: Salama, Y. and Rocha, J. (2022) Aeroacoustic Investigation of Trailing-Edge Finlets. Advances in Aerospace Science and Technology, 7, 1-24. https://doi.org/10.4236/aast.2022.71001

Received: December 20, 2021

Accepted: January 26, 2022

Published: January 29, 2022

Copyright (c) 2022 by author(s) and Scientific Research Publishing Inc. This work is licensed under the Creative Commons Attribution International License (CC BY 4.0).

http://creativecommons.org/licenses/by/4.0/

\begin{abstract}
Wind tunnel testing and embedded large eddy simulations are employed to study the noise reduction of trailing-edge finlets on an airfoil. Trailing-edge finlets are shown to increase the distance between the highly energetic fluid particles and the sharp trailing edge. Experiments were conducted at different angles of attack. Wind tunnel measurements confirm that finlets reduce the broadband noise radiated by the airfoil. Results also reveal that the noise reduction of finlets is dependent on the airfoil angle of attack, and that the highest noise reduction is obtained at the largest angle of attack tested.
\end{abstract}

\section{Keywords}

Wind Tunnel Testing, Noise, Trailing Edges, Finlets, Airfoil Design

\section{Introduction}

With noise levels increasing at the community level, aerodynamic noise reduction has been gaining the attention of the research community. Over the past few decades, noise pollution has increased, disturbing the integrity of natural ecosystems and putting these at risk [1]. Likewise, humans are affected by noise pollution as it impacts their quality of life and puts their mental and physical well-being at risk [2].

In parallel, global warming has led to a universal push towards sustainability, promoting increased interest in renewable energy sources to replace coal and fossil fuels, and reduce greenhouse gas emissions. Harnessing the energy of wind through wind turbines is one of those sources. Despite their many advantages, the noise produced by such turbines is still of the most significant hindrance preventing their widespread use, and the largest contributor to this noise pollution is that generated by the Trailing Edge (TE) of wind turbine blades [3]. For those reasons, TE noise reduction has become of crucial importance for many indus- 
trial sectors.

Researchers and engineers turned to nature to investigate the possibility of having low-noise airfoils. In 1934, R. R. Graham [4] was the first to recognize the potential of using birds as the reference to render modern airplanes more efficient, and specifically identified owls as a biomimicry candidate to achieve silent flight. The wings of owls differ from those of all other groups of birds. Three main noise reduction peculiarities were observed in owl wings, which distinguish them from other birds:

1) In front of every feather (leading-edge), a remarkably stiff comb-like fringe exists;

2) Spanning along the trailing edge of the main wing, a fringe resembling that of a shawl exists;

3) Parts of the upper surface of the wings are covered with fine short feathers.

Soon afterwards, more researchers followed the same path and were drawn to nature looking for inspiration. In 1998, Lilley [5] confirmed the three main noise suppression mechanisms in owl wings previously addressed by Graham [4]. Following the work of these authors, further work has been conducted using the owl as a biomimicry model. The proceeding step was to implement owl wing features in a practical way on solid airfoils to study their efficiency as noise suppressers and their effect on aerodynamic flow properties. Clark et al. [6] designed surface treatments that would replicate the surface-pressure attenuating effects of the downy upper surface of owl wings, in a form suitable for application on an actual airfoil. Afshari et al. [7] investigated the effect of different types of surface treatments on surface pressure fluctuations, eddy convection velocity and spanwise length scale on a flat plate model, where the Boundary Layer $(\mathrm{BL})$ was tripped. The experimental results revealed that having coarser finlet spacing reduces the surface pressure power spectral density at mid to high frequency and increases the spanwise length scale, while finer spacings effectively suppress higher frequency fluctuations with a penalty in the low- to mid-frequency range, and a reduction in the spanwise length scale.

Numerical simulations have also been used to predict far-field noise radiation. In 2000, Manoha, Troff and Sagaut [8] successfully predicted the far-field noise generated by turbulence flowing over the blunt TE of a thick flat plate by coupling a Large Eddy Simulation (LES) with Curle's solution to the Lighthill equation, then the Ffowcs-Williams and Hawkings (FW-H) analogy. Agrawal and Sharma [9] also assessed the effectiveness of biomimicry in reducing aerodynamic noise using LES. The interaction between the wake of a cylindrical rod and a downstream airfoil was simulated to investigate the effect of sinusoidal leading-edge serrations on radiated noise. Tang et al. [10] performed simulations employing LES using the Lighthill-Curle method in an attempt to reveal the variation in the hydrodynamic field and sound source associated with TE serrations on a NACA 0012 airfoil. Zilstra and Johnson [11] demonstrated the ability of LES, combined with the 
FW-H acoustic analogy, to predict the flow field and acoustic results for an SD7037 airfoil at a Re of 43,000 and different angles of attack. Bodling et al. [12] presented a numerical investigation of bio-inspired airfoils finlets. The used geometries were characterized by an array of finlets applied near the TE and aligned with the flow. LES was carried out on a NACA0012 airfoil for two different fence heights. Overall, the method proved to be an effective airfoil self-noise prediction tool at static angles of attack.

Despite the increase in computing power over the last two decades, Large Eddy Simulations remain prohibitively expensive. Because of the impracticality of LES and the need for reliable short-response-time noise prediction methods for industrial design and optimization, some researchers resorted to statistical models based on steady RANS solutions in a sequential CFD/CAA approach. Markus [13] reviewed three different methods based on steady Reynolds-averaged NavierStokes (RANS) solutions to predict noise emitted from airfoils. Validation studies showed decent agreement between the considered methods and results from experiments, a semi-empirical airfoil self-noise prediction code and LES. In another attempt to get accurate noise predictions at a reasonable computational cost, Quéméré and Sagaut [14] presented a novel zonal multi-domain RANS/LES method (also known as Embedded LES or ELES), where the full domain configuration was decomposed into several subdomains that can be treated with either RANS or LES. The same concept was later adopted by Teraccol [15], who investigated using ELES to represent aerodynamic noise sources. The method was applied to a flat plate with a blunt TE and a NACA0012 airfoil. In this approach, zonal LES is only performed close to the main elements responsible for sound generation, while the overall configuration is treated by RANS. The most critical point was the numerical treatment performed at the inlet of the LES domain. CPU time reductions in the order of 40 were obtained and the method was found to be an attractive compromise between accuracy and computational cost. In 2008, Fröhlich and von Terzi [16] presented a generic review of the various ELES approaches along with different interface treatment strategies. The review provided information on how to distinguish between the different methods and advanced the understanding of their inherent limitations as well as the encountered difficulties. Successful simulation results demonstrated the high potential of the approach. Kim et al. [17] used a segregated ELES approach to predict the aeroacoustic and aerodynamic properties of several flatback airfoils at high Re and compared the results to semi-empirical and experimental data. Synthetic turbulence was generated at the RANS/LES interface using the vortex method and far-field acoustics were computed using the FW-H analogy. The obtained frequency spectra of surface pressure fluctuations obtained is in good agreement with experimental measurements at the same observer location and the hybrid RANS-LES method is found to be adequate for predicting aerodynamic noise generation by vortical flow in the vicinity of a blunt TE airfoil over a range of frequencies. Lane, Croaker and Ding [18] tested and implemented ELES for the prediction of TE noise due 
to flow around a NACA 0012 airfoil. The obtained results were compared to a full LES simulation and to experimental data. Both simulations used the same mesh resolution and the same wall-modeled LES approach. For ELES, the mesh size was only about 13 million cells, compared to 40 million cells for the full LES. It was found that the results of both simulations were in good agreement. The ELES approach resulted in saving 55\% of the computational cost of a full LES. Zuo et al. [19] performed flow simulations using ELES to analyze the aerodynamic and noise characteristics of a serrated-TE NACA0018 airfoil at a Re of 160,000 and an Angle of Attack (AOA) of 6 degrees. Two airfoils having the same serration wavelength and different serration amplitudes were considered and compared to a straight TE case. Predictions based on the FW-H acoustic analogy showed that longer serrations are more effective in decreasing the overall sound pressure levels.

The main objective of the current study is to investigate the use of LES as a relatively fast aeroacoustic and aerodynamic framework for the prediction of noise (i.e., as a tool that could be used in the preliminary stage of design), hence possibly reducing the computational cost of such investigations at this phase of design. This study also aims at improving the overall knowledge on the working principles of the effect of bio-inspired designs on flow and fair-field noise. The used ELES configuration, where the LES region only partially covers the airfoil chord- length, hasn't been used to investigate bio-inspired finlets yet. ELES is adopted to study the flow field around a flat-TE NACA0012 airfoil and a finned-TE airfoil. The far-field noise is computed using the Ffowcs Williams-Hawkings (FW-H) model. The chord-based Reynold's number, $R e_{\odot}$ is approximately 500,000. Experimental testing is then conducted to evaluate the effect of the airfoil AOA on the noise reduction by the finlets design.

The paper is structured as follows: Section 2.1 presents the numerical methodology with the governing LES equations, while Sections 2.2 and 2.3 describe the hybrid RANS/LES interface treatment and the FW-H aeroacoustic analogy, respectively. Section 3 presents the flow configuration (Section 3.1), the computational mesh (Section 3.2), and the experimental setup (Section 3.3). Results are discussed in Section 4.

\section{Numerical Methods}

\subsection{Governing Equations}

The governing equations used in the current study, termed the spatially-filtered Navier-Stokes equations, are obtained by applying a low pass filter on the time dependent Navier-Stokes equations in the physical space. The simulations were carried out using the commercial software ANSYS Fluent. The flow is assumed incompressible. In order to increase efficiency, the filter width is the same size as the mesh spacing used in the computational domain. The resulting equations describe the dynamics of large eddies [20] [21]. Field variables, such as pressure and velocity, are defined by their convolution with a filter function over the fluid do- 
main, as following:

$$
\bar{\phi}(x)=\int_{D} \phi\left(x^{\prime}\right) G\left(x, x^{\prime}\right) \mathrm{d} x^{\prime}
$$

where: $D$ is the fluid domain and $G$ is the filtering function. The overbar indicates spatial filtering and not temporal averaging. After applying the filter to the mass and momentum conservation equations, the NS equations become:

$$
\begin{gathered}
\frac{\partial \rho}{\partial t}+\frac{\partial}{\partial x_{i}}\left(\rho \bar{u}_{i}\right)=0 \\
\frac{\partial}{\partial t}\left(\rho \bar{u}_{i}\right)+\frac{\partial}{\partial x_{j}}\left(\rho \bar{u}_{i} \bar{u}_{j}\right)=\frac{\partial}{\partial x_{j}}\left(\sigma_{i j}\right)-\frac{\partial \bar{p}}{\partial x_{i}}-\frac{\partial \tau_{i j}}{\partial x_{j}}
\end{gathered}
$$

In order to obtain a close system of equations, the unknown SGS stresses are modeled by applying the Boussinesq eddy viscosity hypothesis [22], thus computing the sub-grid-scale turbulent stresses from:

$$
\tau_{i j}-\frac{1}{3} \tau_{k k} \delta_{i j}=-2 \mu_{t} \bar{S}_{i j}
$$

where: $\mu_{t}$ is the subgrid-scale turbulent viscosity and $\tau_{k k}$ is the isotropic part of the SGS. The latter part is not modeled as it is added to the filtered static pressure term. $S_{i j}$ is the strain-rate tensor of the resolved scale calculated from Equation (5) using the filtered velocity components.

$$
\bar{S}_{i j}=\frac{1}{2}\left(\frac{\partial \bar{u}_{i}}{\partial x_{j}}+\frac{\partial \bar{u}_{j}}{\partial x_{i}}\right)
$$

Using the Wall Adapting Local Eddy Viscosity (WALE) model [23], $\mu_{t}$ is modeled as:

$$
\mu_{t}=\rho L_{s}^{2} \frac{\left(S_{i j}^{d} S_{i j}^{d}\right)^{\frac{3}{2}}}{\left(\bar{S}_{i j} \bar{S}_{i j}\right)^{\frac{5}{2}}\left(S_{i j}^{d} S_{i j}^{d}\right)^{\frac{5}{4}}}
$$

where: $L_{s}$ the mixing length of the sub-grid scale, and $S_{i j}^{d}$, which is a function of the strain and rotation rate tensors, are defined in Equations (7) and (8) as:

$$
\begin{gathered}
L_{s}=\min \left(\kappa d, C_{w} V^{\frac{1}{3}}\right) \\
S_{i j}^{d}=\frac{1}{2}\left(\bar{g}_{i j}^{2}+\bar{g}_{j i}^{2}\right)-\frac{1}{3} \delta_{i j} \bar{g}_{k k}^{2}
\end{gathered}
$$

with $g_{i j}$ defined as:

$$
\bar{g}_{i j}=\frac{\partial \bar{u}_{i}}{\partial x_{j}}
$$

where: $d$ is the distance to the closest wall, $V$ is the volume of the computational cell, $\kappa=0.41$ is the von Kármán constant, and $C_{W}=0.325$ is the WALE constant. Values of $d$ and $V$ are determined by the ANSYS Fluent code for each mesh element. The von Kármán constant (defined as $\kappa=y / I$ ) is one of the few funda- 
mental constants in fluid mechanics governing turbulent flows, and represents the ratio between mixing length, $l$, and the vertical distance in the flow field, $y$. Using an energy argument and mathematical symmetry it is argued that the von Kármán's constant is $\kappa=0.414$ (with derivable corrections for specific applications) [24]. As shown in [25], the best estimate for the von Kármán constant is found to be $0.40 \pm 0.02$. In the present simulation, $\kappa=0.41$ was used, as specified in the ANSYS Fluent manual for external flows [20]. The WALE constant is used to estimate the mixing length of the subgrid scale, which is necessary to evaluate the unresolved (subgrid) turbulence of the flow in LES. Intensive validation during a European Union research project [23] has shown consistently superior results using $C_{W}=0.325$ [26]. In addition, as described in the ANSYS Fluent manual [20], a value of the WALE constant of 0.325 has shown to yield satisfactory results for a wide range of flow; hence, this value was used in the current simulation.

\subsection{RANS/LES Interface}

In the present study and approach used, the entire domain is decomposed into clearly identifiable regions for RANS and LES before the simulation is started. This is usually referred to as segregated modeling. The goal is to use each model where it is best suited. The flow is initialized using RANS equations, which provide stationary field statistics, and LES re-solves the unsteady high-resolution perturbations near the TE, where it is needed. The main difficulty is defining proper interface conditions, seeing that inappropriate coupling could lead to results' contamination in the LES or RANS subdomains. At the inflow interface, mass, momentum and energy are convected into the LES subdomain from the RANS region. The latter provides mean values which are to be coupled with the LES data. To obtain correct LES results, fluctuations must be provided at the interface and added to the mean flow computed by RANS. These fluctuations can be real, provided by precursor simulations or databases of similar flows, or synthetic, provided by Fourier modes, digital filters, random vortices, etc. The goal is to make the imposed fluctuation as close as possible to those present in a real physical flow.

The Vortex Method [27] was chosen as a means of adding artificial resolved turbulence at the RANS/LES interface. In this approach, a fluctuating vorticity field is added to the mean flow, consequently creating perturbations similar in behavior to realistic ones. The VM is based on the Biot-Savart law and the 2D evolution equation of vorticity. Vortex points, or particles, are distributed over the inlet interface perpendicular to the streamwise direction and are randomly convected, carrying information about the vorticity field. The amount of vorticity carried by a given particle " $i$ " is represented by the circulation $\Gamma$ according to Equation (11), and the assumed spatial distribution is given by Equation (12), such that:

$$
\omega(\boldsymbol{x}, t)=\sum_{i=1}^{N} \Gamma_{i}(t) \eta\left(\left|\boldsymbol{x}-\boldsymbol{x}_{i}\right|, t\right)
$$




$$
\begin{gathered}
\Gamma_{i}(x, y)=4 \sqrt{\left(\frac{\pi A k(x, y)}{3 N(2 \ln (3)-3 \ln (2))}\right)} \\
\eta(x)=\frac{1}{2 \pi \sigma^{2}}\left(2 \mathrm{e}^{\frac{-|x|^{2}}{2 \sigma^{2}}}-1\right) 2 \mathrm{e}^{\frac{-|x|^{2}}{2 \sigma^{2}}}
\end{gathered}
$$

where: $N$ is the number of vortex points, $A$ is the inlet section area, $k$ is the turbulence kinetic energy and $\sigma$ controls the size of the vortex particles. The resulting discretization for the velocity field is given by:

$$
\boldsymbol{u}(\boldsymbol{x})=\frac{1}{2 \pi} \sum_{i=1}^{N} \Gamma_{i} \frac{\left(\boldsymbol{x}_{i}-\boldsymbol{x}\right) \times \boldsymbol{z}}{\left|\boldsymbol{x}-\boldsymbol{x}_{i}^{\prime}\right|^{2}}\left(1-\mathrm{e}^{\frac{\left|\boldsymbol{x}-\boldsymbol{x}^{\prime}\right|^{2}}{2 \sigma^{2}}}\right) \mathrm{e}^{-\frac{\left|\boldsymbol{x}-\boldsymbol{x}^{\prime}\right|^{2}}{2 \sigma^{2}}}
$$

in which $z$ is a unit vector in the streamwise direction and $x_{i}$ is the location of the $i$-th vortex particle. The value of $\sigma$ is calculated from a known profile of mean turbulence kinetic energy and mean dissipation rate at the inlet, such that:

$$
\sigma=\frac{c k^{\frac{3}{2}}}{2 \epsilon}
$$

in which $c=C \mu^{3 / 4}=0.16$, with $C \mu=0.09$ as shown in [27]. Using the ANSYS Fluent code, the minimum value of $\sigma$ is determined by the local mesh size to ensure that the vortices will always belong to the resolved scale. The sign of the circulation of each vortex is randomly changed every characteristic time scale, which is the time needed for a $2 \mathrm{D}$ vortex to travel $\mathrm{n}$ times its mean characteristic $2 \mathrm{D}$ size in the boundary normal direction, where $n$ is set to equal 100 from numerical testing.

\subsection{FW-H Aeroacoustic Analogy}

In order to overcome the prohibitive cost of directly resolving the pressure fluctuations responsible for noise in the far-field, a method based on Lighthill's acoustic analogy [28] is used. In this approach, the nearfield flow is computed using the appropriate governing equations of ELES, and the far-field noise is predicted with the aid of an analytically de-rived integral solution to the wave equation. The acoustic analogy decouples sound generation from its propagation, thus allowing the separation of the flow solution from the acoustic analysis and the extraction of acoustic sources from the CFD domain.

The Ffowcs Williams and Hawkings (FW-H) formulation [29] adopts the most gen-eral form of Lighthill's acoustic analogy. The FW-H equation [29] [30] is nothing but an in-homogeneous wave equation derived by manipulating the continuity and Navier-Stokes equation. The FW-H equation can be expressed as:

$$
\begin{aligned}
\frac{1}{a_{\infty}^{2}} \frac{\partial^{2} p^{\prime}}{\partial t^{2}}-\nabla^{2} p^{\prime}= & \frac{\partial}{\partial t}\left\{\left[\rho_{\infty} v_{n}+\rho\left(u_{n}-v_{n}\right)\right] \delta(f)\right\} \\
& -\frac{\partial}{\partial x_{i}}\left\{\left[P_{i j} n_{j}+\rho u_{i}\left(u_{n}-v_{n}\right)\right] \delta(f)\right\}+\frac{\partial^{2}}{\partial x_{i} \partial x_{j}}\left\{T_{i j} H(f)\right\}
\end{aligned}
$$




$$
T_{i j}=\rho u_{i} u_{j}+P_{i j}-a_{\infty}^{2}\left(\rho-\rho_{\infty}\right) \delta_{i j}
$$

where: $p^{\prime}=p-p_{\infty}$ is the sound pressure at the far-field, $u_{i}$ is the fluid velocity component in the $x_{i}$ direction, $u_{n}$ is the velocity component normal to the surface $f=0, v_{i}$ is the surface velocity component in the $x_{i}$ direction, $v_{n}$ is the surface velocity component normal to the surface, $\delta(f)$ is the Dirac delta function and $H(f)$ is the Heaviside function. The subscript " $\infty$ " denotes freestream parameters. The $f=0$ surface is a mathematical surface representing the source surface. $n_{i}$ is a unit vector normal pointing towards the exterior region of the source $(f>0), a_{\infty}$ is the speed of the sound at the far field, $T_{i j}$ is the Lighthill stress tensor defined in Equation (17), and $P_{i j}$ is the compressive stress tensor. The first term on the right hand side of Equation (16) represents the monopole or thickness source, modeling the sound generated by the displacement of a fluid as a body passes through it. The second term is the dipole or loading source, resulting from the unsteadiness of the forces acting on the body's surface. The third term is the quadrupole source term, representing the non-linear fluctuations in the local sound speed and fluid velocity near the body surface. Monopole and dipole sources are dominant in low Mach number flows. By integrating Equation (16) assuming free-space flow and no obstacles between the sound source and receiver, a full solution consisting of surface and volume integrals is obtained. In the present case, the volume integral is neglected as it is only significant in high Mach number flows. Thus, far-field sound pressure can be expressed as:

$$
p^{\prime}(x, t)=p_{T}^{\prime}(x, t)+p_{L}^{\prime}(x, t)
$$

in which:

$$
\begin{gathered}
4 \pi p_{T}^{\prime}(x, t)=\int_{f=0}\left[\frac{\rho_{\infty}\left(\dot{U}_{n}+U_{\dot{n}}\right)}{r\left(1-M_{r}\right)^{2}}\right] \mathrm{d} S \\
+\int_{f=0}\left[\frac{\rho_{\infty} U_{n}\left\{r \dot{M}_{r}+a_{\infty}\left(M_{r}-M^{2}\right)\right\}}{r^{2}\left(1-M_{r}\right)^{3}}\right] \mathrm{d} S \\
4 \pi p_{L}^{\prime}(x, t)=\frac{1}{a_{\infty}} \int_{f=0}\left[\frac{\dot{L}_{r}}{r\left(1-M_{r}\right)^{2}}\right] \mathrm{d} S+\int_{f=0}\left[\frac{L_{r}-L_{M}}{r^{2}\left(1-M_{r}\right)^{2}}\right] \mathrm{d} S \\
+\frac{1}{a_{\infty}} \int_{f=0}\left[\frac{L_{r}\left\{r \dot{M}_{r}+a_{\infty}\left(M_{r}-M^{2}\right)\right\}}{r^{2}\left(1-M_{r}\right)^{3}}\right] \mathrm{d} S \\
U_{i}=v_{i}+\frac{\rho}{\rho_{\infty}}\left(u_{i}-v_{i}\right) \\
L_{i}=P_{i j} \hat{n}_{j}+\rho u_{i}\left(u_{n}-v_{n}\right)
\end{gathered}
$$

A dot over a variable indicates the source-time derivative of that variable, while the subscripts $n, r$ and $M$ denote the dot product with the unit normal vector, the unit radiation vector and surface velocity vector normalized by the speed of 
sound, respectively.

\section{Flow Configuration, Computational and Experimental Setups}

\subsection{Flow Configuration}

The airfoil selected for the present simulation is a NACA0012 symmetric airfoil, at zero angle of attack to isolate the effect of lift generation on the analysis. The chord length of the airfoils $c$ is $0.3 \mathrm{~m}$. The airfoil is placed in a square $10 c \times 10 c$ domain, which was sufficient to provide convergence of results. The flow domain is divided into two regions as seen in Figure 1. RANS equations are employed in a coarse RANS domain, while LES equations are employed in a refined LES region near the TE. It's important to note that only the noise radiated by the flow within the LES region is predicted in the numerical simulations. Since the presented work is focused on TE noise predictions, it is reasonable to neglect the noise generated by other airfoil sections, such as the leading edge. $s$ is the span of the flow domain. Two embedded configurations were tested. For cases C1.1 and C1.2, the LES domains in the streamwise direction extend from $\frac{X}{C}=0.5$ and $\frac{x}{c}=0.7$, respectively, to $1 c$ downstream of the TE. The letter ' $\mathrm{C}$ ' stands for computational. The origin is defined at the airfoil leading edge. In the transverse direction, the LES domain extends $0.25 c$ above and below the airfoil. TE finlets are also tested. The thickness of the finlets, $t$, is $1 \mathrm{~mm}$ and the spacing between each two consecutive finlets, $S$, is $3.9 \mathrm{~mm}$. The finlets do not extend past the airfoil TE, and their LE falls at $\frac{x}{c}=0.73$. The maximum height of the finlets $(H)$, as measured from the airfoil surface, is $8 \mathrm{~mm}$, and is reached at $\frac{x}{c}=0.975$. The design parameters are inspired by the work of Bodling et al. [12] and Shi et al. [31]. Figure 2 shows a general model of TE finlets. Table 1 summarizes the geometric parameters of the simulated cases. Computations are carried out at a free stream velocity $u_{\infty}=24 \mathrm{~m} / \mathrm{s}$ and a free stream Mach number $M_{\infty}=0.071$, resulting in a chord-based Reynolds number, $R e_{c}=\rho u_{\infty} \frac{D}{\mu}$, of approximately

\section{RANS Zone}

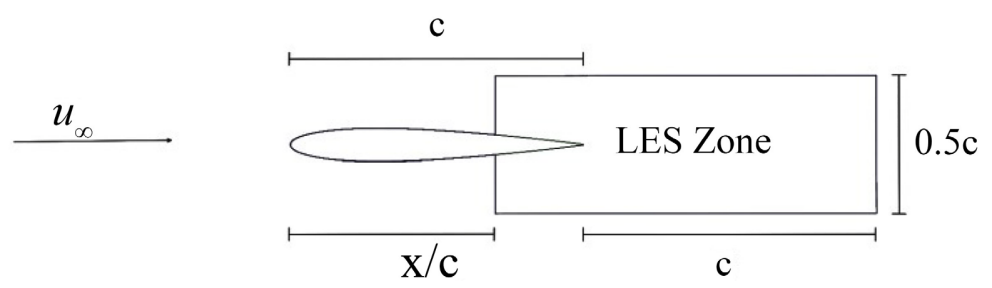

Figure 1. Schematic illustration of the segregated modeling domains. 
Table 1. Geometric parameters of simulated airfoils.

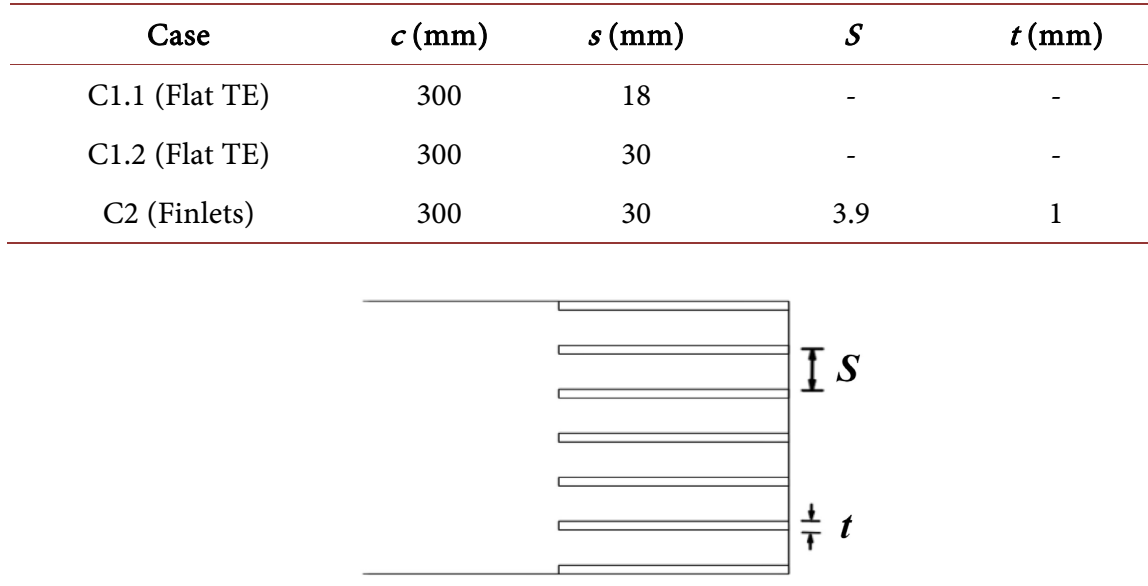

(a)

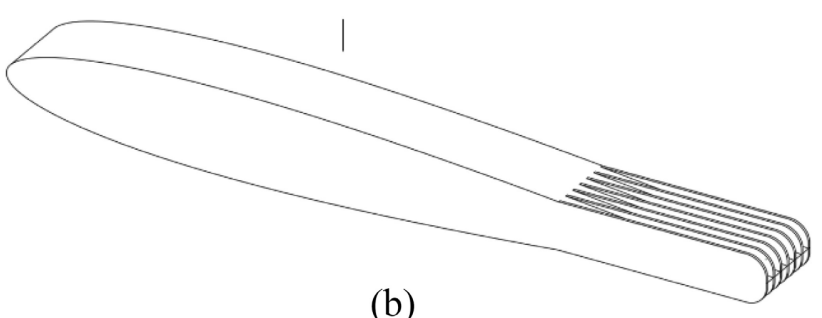

(b)

Figure 2. General model of TE finlets, not to scale. (a) Top view; (b) Isometric view.

500,000 , where $\rho$ is the fluid density, $\mu$ is the dynamic viscosity and $D$ is the characteristic length, which is the airfoil chord in this case.

\subsection{Computational Mesh}

A predominantly hexahedral mesh is generated following the cartesian cut-cell method (Figure 3). This meshing technique, which has received a significant development in recent years [32], was found ideal for the current study seeing that it results in a smaller number of elements for the same resolution compared to other methods, thus significantly reducing the simulation time. In addition, the resulting elements are characterized by their high orthogonal quality and low skewness, which minimizes truncation errors [32] [33]. Element size is restricted to $25.6 \mathrm{~mm}$ in the coarse RANS zone, $0.8 \mathrm{~mm}$ in the refined LES zone, and 0.2 $\mathrm{mm}$ on the airfoil surface in the vicinity of the TE. Elements in the airfoil wake of the RANS zone have a size of $5 \mathrm{~mm}$. The grid resolution in terms of wall-normal units is defined by $\Delta x^{+}=\frac{u_{\tau} \Delta x}{v}, \Delta y^{+}=\frac{u_{\tau} \Delta y}{v}$ and $\Delta z^{+}=\frac{u_{\tau} \Delta z}{v}$ where $u_{\tau}$ is the frictional velocity and $v$ is the kinematic viscosity. 40 inflation layers (Figure $3(c))$ are generated around the airfoil with the thickness of the first layer set to $7.6 \times 10^{-3} \mathrm{~mm}$ and a growth factor of 1.08 , thus ensuring $y^{+}<0.5$ everywhere on the airfoil surface (Figure 4), at least three layers in the viscous sublayer and overall accurate boundary layer resolution. Table 2 lists mesh properties for all 


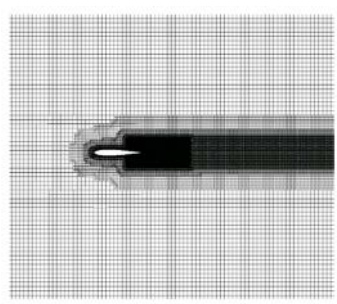

(a)

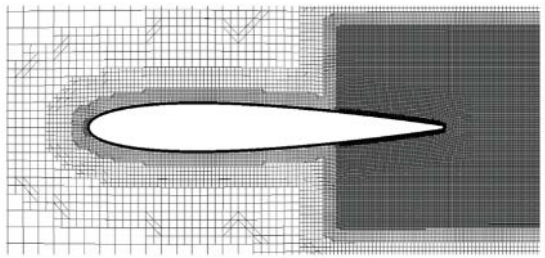

(b)

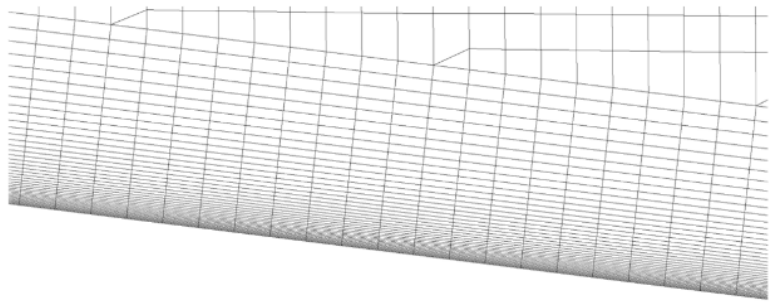

(c)

Figure 3. Computational mesh setup. (a) Mesh overview; (b) Mesh near airfoil surface; (c) Inflation layers around airfoil.
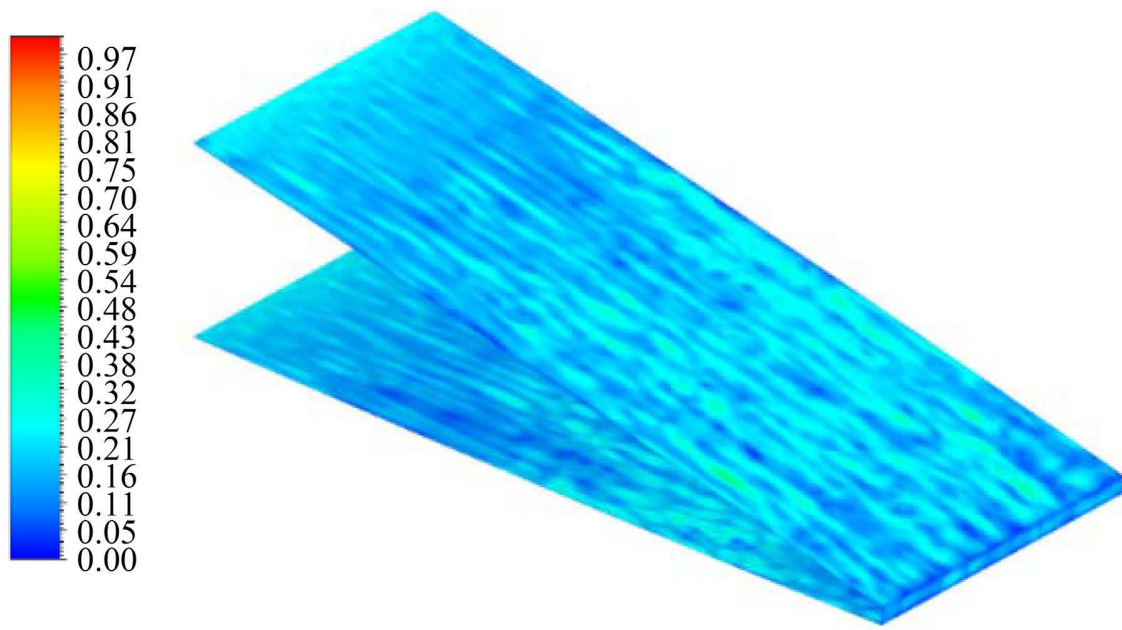

Figure 4. Instantaneous $y^{+}$distribution.

Table 2. Number of elements in each computational mesh.

\begin{tabular}{cccc}
\hline Case & RANS & LES & Total \\
\hline C1.1 & 456,353 & $4,586,780$ & $5,043,133$ \\
C1.2 & 976,300 & $5,690,368$ & $6,666,668$ \\
C2 & 976,300 & $7,772,301$ & $8,748,601$ \\
\hline
\end{tabular}

simulated cases. The chosen computational grid has a maximum resolution $\Delta x_{\max }^{+} \leq 20$ and $\Delta z_{\max }^{+} \leq 20$ in the streamwise and spanwise directions, respectively [34] [35]. For verification, a third case was is simulated using two different meshes to investigate the effect of the mesh on the predicted noise. A steady-state mesh convergence study was carried out by progressively refining the mesh, creat- 
ing three meshes having 6,666,668 elements, 7,606,083 elements and 9,011,531 elements, respectively. The values of integrated output parameters, such as lift and drag coefficients, were compared and the maximum error is found to be less than $0.4 \%$, demonstrating mesh convergence. Furthermore, the first two meshes were carried over for a transient simulation analysis. The lift-history coefficients were evaluated for each mesh at every time step and their RMS values were computed. Both meshes yield the same lift-coefficient RMS value, $c_{L_{\mathrm{RMS}}}=0.0013$. Consistent results in terms of integrated flow parameters for both steady-state and transient simulations, are a strong indication of the convergence of the used computational mesh, i.e., the mesh directly resolves enough flow structures for the results not to change with mesh refinement.

The boundary conditions used are demonstrated in Figure 5. A velocity inlet boundary condition is specified at the domain entrance, where $u_{\infty}=24 \mathrm{~m} / \mathrm{s}$. Periodic Boundary Conditions (PBCs) are applied on the right and left side walls of the domain in the spanwise direction to allow the flow to develop naturally. No-slip boundary conditions are applied on the airfoil surface and a zero gauge-pressure outlet boundary condition is used. The inlet turbulence is set to $0.3 \%$. The SIMPLE pressure-velocity coupling scheme is used. All results are second order accurate in time and space. The flow is initialized using the $k$ - $\omega$ SST model developed by Menter [36], as it gives accurate separation predictions for external flows. The VM is then used to inject turbulence at the RANS/LES interface and the simulation is run for $4 T_{\mathrm{TF}}$ "Through-flow time" to obtain a fully developed flow, where $T_{\mathrm{TF}}=\frac{L_{\mathrm{CFD}}}{u_{\infty}}$ [37]. $L_{\mathrm{CFD}}$ is the LES domain length in the streamwise direction.

WALE Subgrid-Scale (SGS) modelling is employed in the LES region as it is designed to return correct asymptotic wall behavior for wall-bounded flows [20].

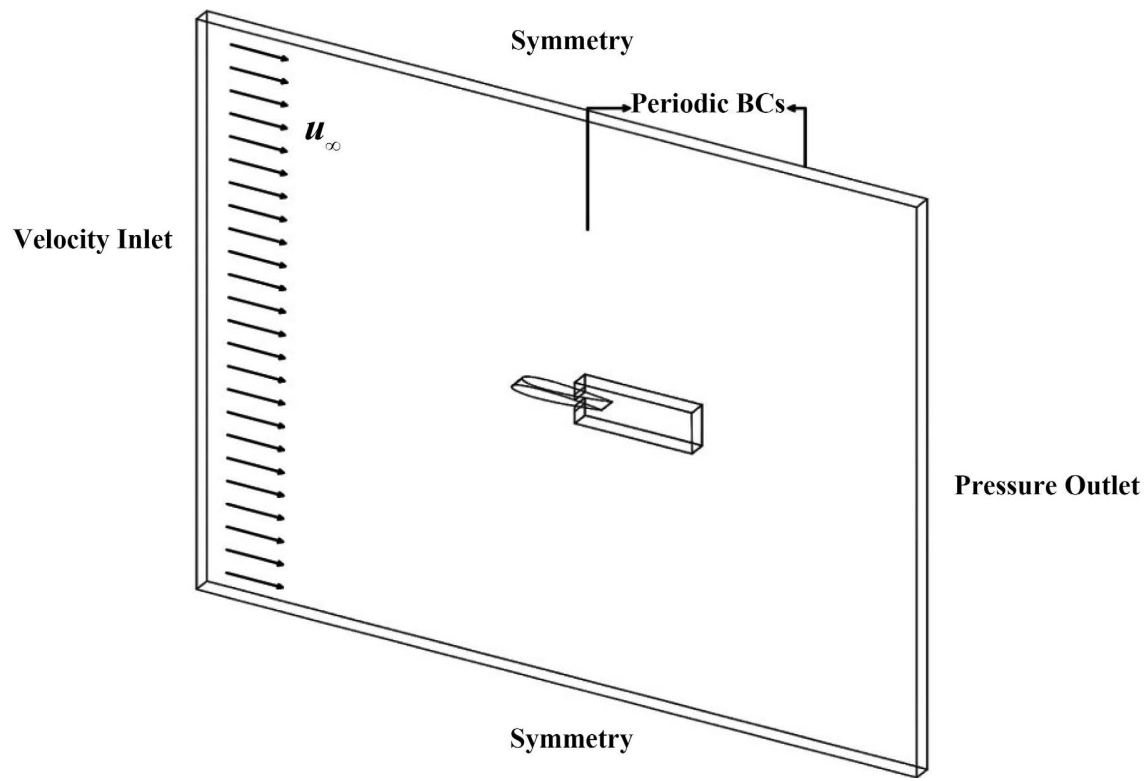

Figure 5. Boundary conditions. 
The time step $d t=1.2 \times 10^{-5}$ seconds. With these values, the Courant-FriedrichsLewy (CFL) number achieved is $\leq 1$ everywhere in the domain, meaning the flow particles don't travel more than the length of one mesh element every time step. Residuals are reduced by three orders of magnitude each time step. Lastly, acoustic data is gathered for $3 T_{\mathrm{TF}}$. All convergence residuals are set to $10^{-5}$. Pressure and velocity monitoring points were placed in the airfoil wake and statistical convergence is achieved. Statistical convergence is also achieved for the coefficients of lift and drag. All simulations are carried out using the commercial CFD software FLUENT 2019R3 and run on Intel Xeon L5410 $2.33 \mathrm{GHz}$ platform of 60 cores.

\subsection{Experimental Setup}

Experiments were conducted in the medium-speed, subsonic, closed-loop wind tunnel at Carleton University (Figure 6). A series of turbulence grids precede a 9:1 contraction, which reduces the turbulence intensity levels in the center of the test section to less than $0.27 \%$. The tunnel has a removable, rectangular test section along with the surrounding anechoic chambers was completed to be used for aeroacoustic testing. This test section is a $0.78 \mathrm{~m} \times 0.51 \mathrm{~m}$ rectangular section, $1.83 \mathrm{~m}$ long. The upper and lower walls of the test section are each composed of two aluminum sheet panels and contain hardware (circle aluminum material) for the vertical mounting of a two-dimensional airfoil in the midway, and $0.45 \mathrm{~m}$ from the upstream end of the test section [38] [39].

The airfoil wing is mounted vertically in the test section (Figure 7) with its leading edge (at zero AOA) $0.45 \mathrm{~m}$ downstream of the test section entrance. The airfoil under investigation is a NACA0012 airfoil with a finned TE serration, as shown in Figure 7. The chord length of the airfoil is $300 \mathrm{~mm}$, and the width is $510 \mathrm{~mm}$. Between the leading-edge $\left(\frac{x}{c}=0\right)$, and $\frac{x}{c}=0.73 \mathrm{~mm}$, the original

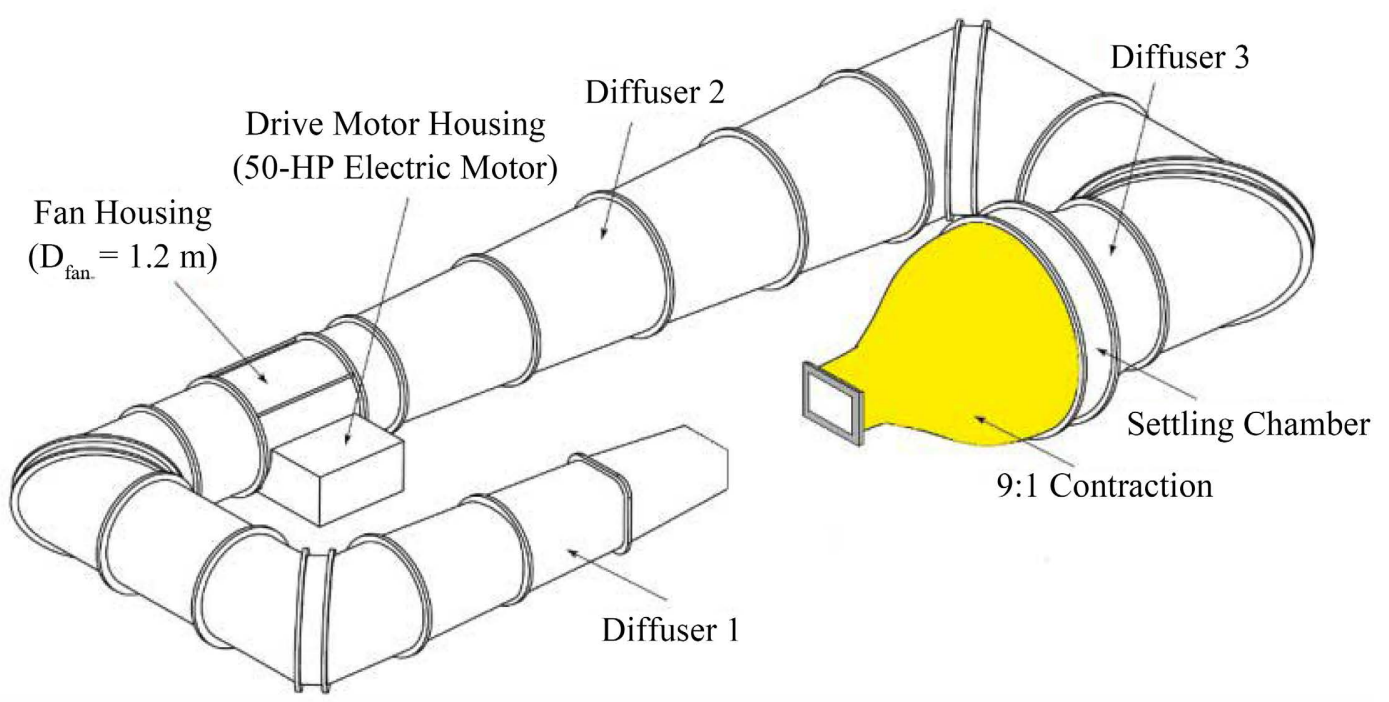

Figure 6. Wind tunnel schematic. 


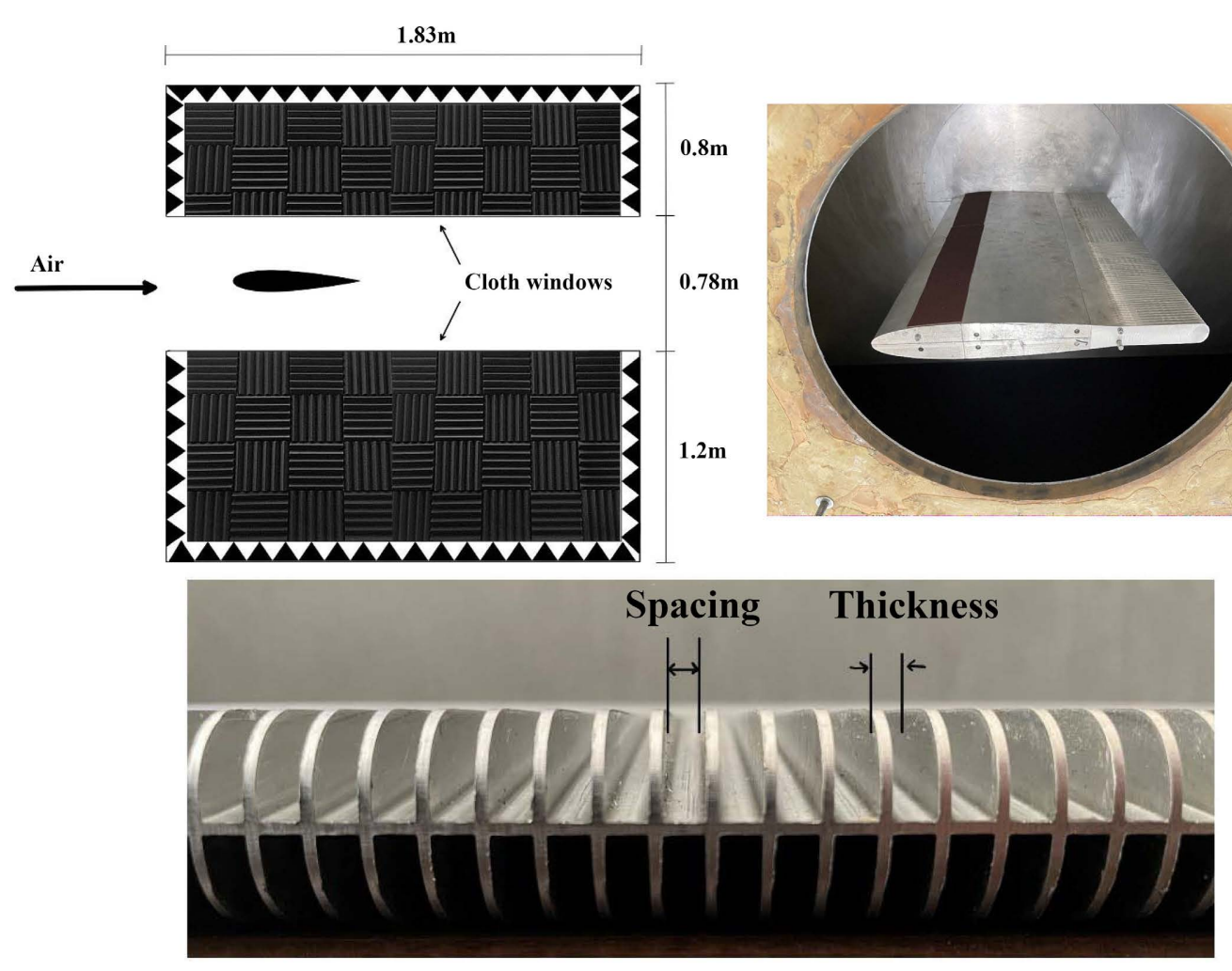

Figure 7. Test section setup and aifoil mounting (top) and finned-TE airfoil (bottom).

NACA0012 airfoil profile is unmodified, where $x$ is the streamwise direction. Further downstream, $0.73 \leq \frac{x}{c} \leq 1.0$, is a section that can be removed and replaced by either an unmodified or modified TE profile. Once attached, the TE section forms a continuous profile giving the appearance that the serrations are cut into the main body of the NACA0012 airfoil. Typical parameters including the finlets height, $H$, and spacing, $S$, are defined as specified in Figure 2. The letter "E" stands for experimental. Far-field noise measurements in the mid-span were performed by a Brüel \& Kjær microphone, which is installed at a distance of 1.4 $\mathrm{m}$ for an observer angle $\alpha=90^{\circ}$. The analysis was carried out between $100 \mathrm{~Hz}$ and $5 \mathrm{kHz}$.

\section{Results}

\subsection{Surface Pressure}

The pressure coefficient distribution around the airfoil is an important parameter since it influences the lift coefficient and the development of the boundary layer [40] [41]. In addition, the BL is responsible for the majority of the generated sound. $C_{p}$ distributions for cases $\mathrm{C} 1.1$ and $\mathrm{C} 1.2$ are computed for validation and compared against experimental results obtained by Lee and Kang [42] for a NACA 0012 airfoil at a $R e=600,000$, and full LES results published by Marsden, Bogey and Bailly [43] at $R e_{c}=500000$ (Figure 8). Excellent agreement is found between the computational and experimental surface pressure results. Of 


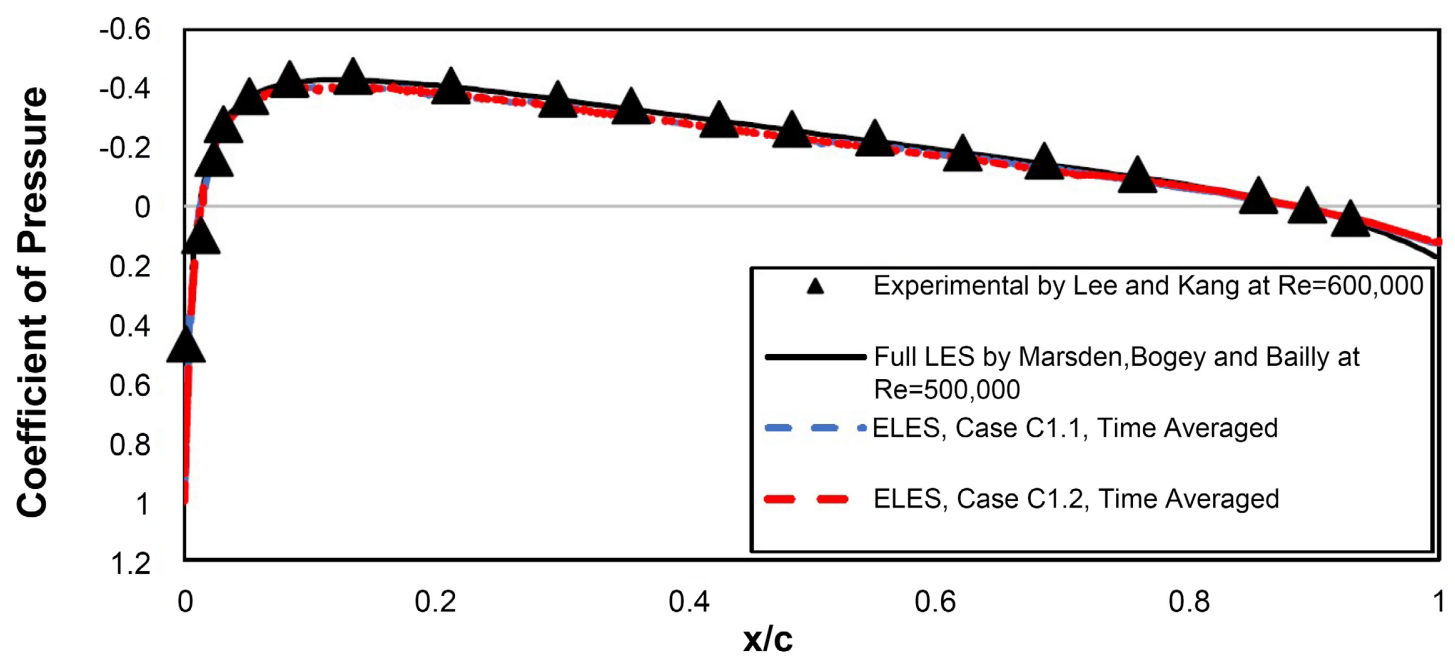

Figure 8. $C_{p}$ distribution on airfoil surface.

importance is the fact that from $\frac{x}{c}=0.15$ down to the TE, the boundary layer is subject to an adverse pressure gradient. Both cases C1.1 and C1.2 are validated against existing literature. The LES domain in C1.1 is longer in the streamwise direction as it starts at a $\frac{X}{C}=0.5$, while it starts at $\frac{X}{C}=0.7$ in C1.2 (see Table 1 and Table 2). Even though both configurations yield acceptable results, the configuration of case C1.2 is chosen for the succeeding simulations as the LES domain covers a larger span.

\subsection{Wake Characteristcs}

By calculating the coefficient of lift, $c_{L}=\frac{L}{0.5 * \rho v^{2} A}$, for every timestep of flow simulation, the lift-coefficient history can be plotted (Figure 9). $L$ is defined as the lift force and $A$ is the airfoil area. The lift-coefficient history is commonly used as an indicator of statistical convergence in transient simulations. Furthermore, it's a non-dimensional representation of the fluctuating forces acting normal to the airfoil surface due to the turbulence of air flow. All axes are kept constant and aligned for the sake of clarity and comparison. For case C1.2, the lift monitor is random and irregular, characterized by a relatively small amplitude. The lift variation behavior in C2 is similar to C1.2. Figure 10 shows the instantaneous flow fields in the airfoil wake in term of iso-surfaces of the Q-criterion, which is defined as the second invariant of the instantaneous velocity gradient tensor [44]. The iso-surfaces are used to identify and portray the turbulent coherent structures of the wake, which are inherently three-dimensional. The iso-surfaces are colored by the spanwise vorticity, $\omega_{z}$, and demonstrate how the wake behavior changes as standard serrations are introduced then their geometrical parameters modified. For the case of a flat TE (C1.2), the wake is non-uniform and has almost no observable coherent structures. For $\mathrm{C} 2$, the wake looks similar and 

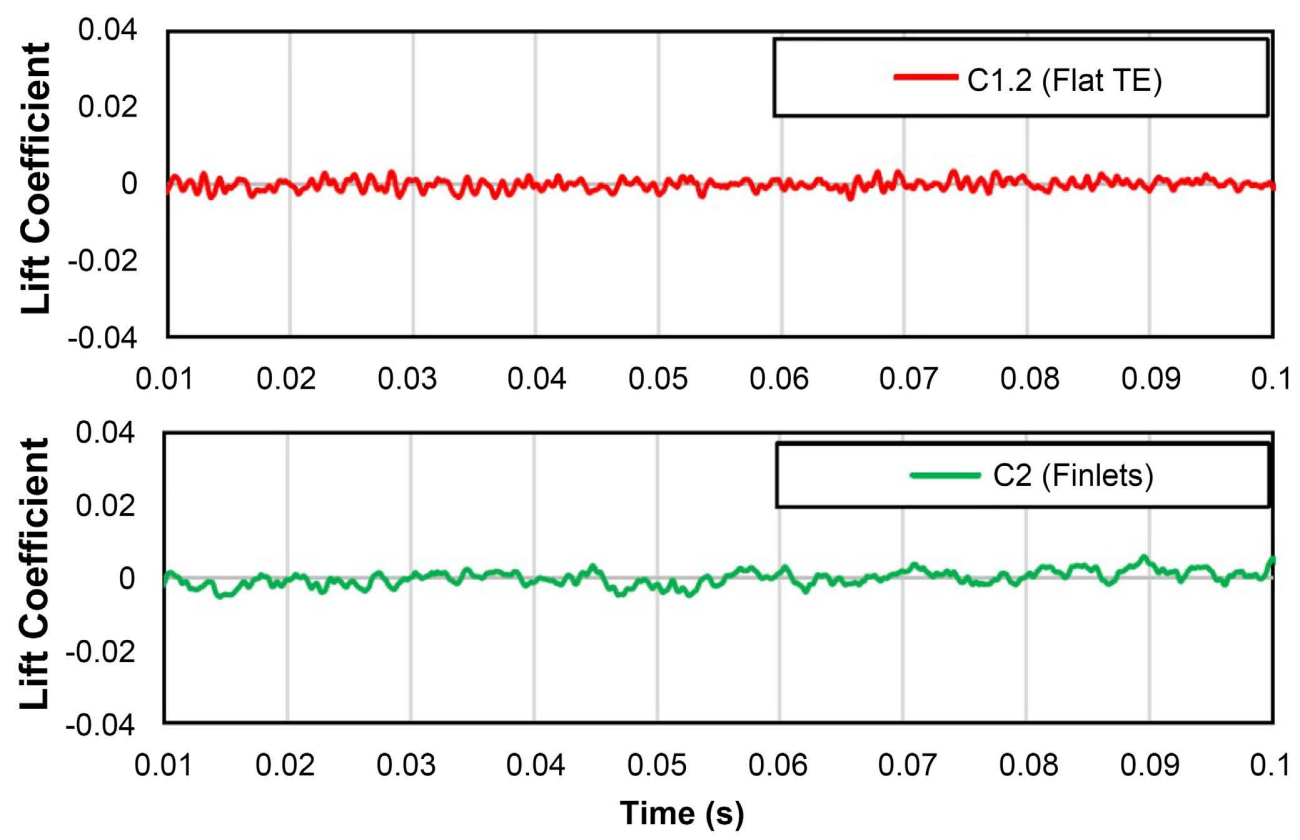

Figure 9. Lift coefficient history.

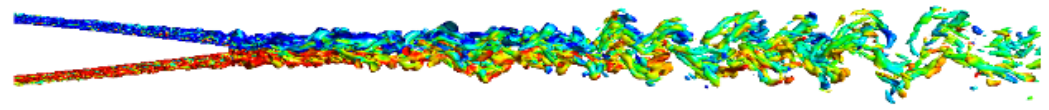

(a)

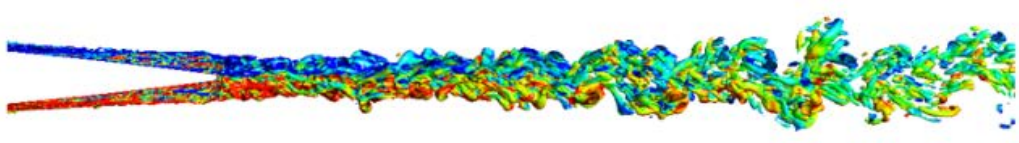

(b)

Figure 10. Instantaneous Q-Criterion colored by $\omega_{z}$. (a) C1.2; (b) C2.

no vortex shedding is observed. The effect of finlets on the instantaneous Turbulence Kinetic Energy (TKE) distribution is shown in Figure 11. Air is channeled between the finlets; the initially undisturbed boundary layer is deformed and decorrelated, and the highly energetic fluid particles are kept away from the airfoil surface, thus decreasing the efficiency of acoustic scattering [45] [46].

\subsection{Far-Field Noise}

The FW-H aeroacoustic analogy [29] is used to compute the radiated far-field noise for the computational cases $\mathrm{C} 1.2$ and $\mathrm{C} 2$. In order to keep the computational cost reasonable, the span of the simulation domains is kept smaller than that of the experimental testing. Acoustic data is sampled every two flow-timesteps and data sampling is performed for $3 T_{\text {TF }}$ after the flow is fully developed, resulting in a sampling frequency of $41.67 \mathrm{kHz}$ and a frequency resolution of $28.4 \mathrm{~Hz}$, where the frequency resolution is defined as the inverse of the sampling period. Pressure fluctuations are propagated to receivers placed midspan at a distance of 


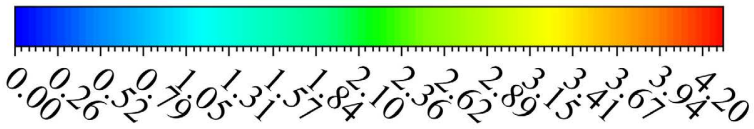

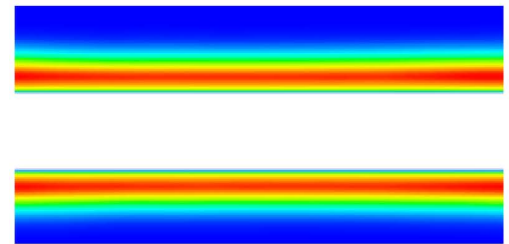

(a)
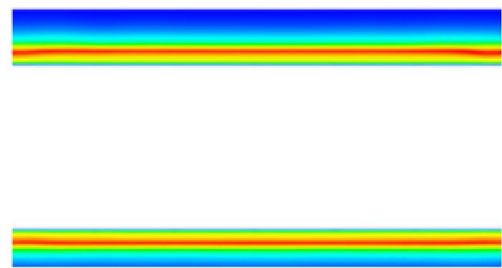

(c)

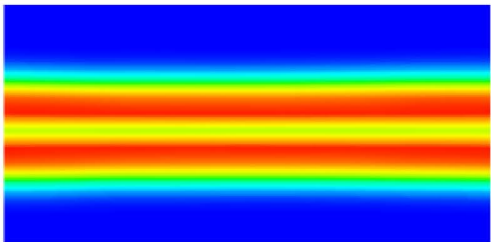

(b)
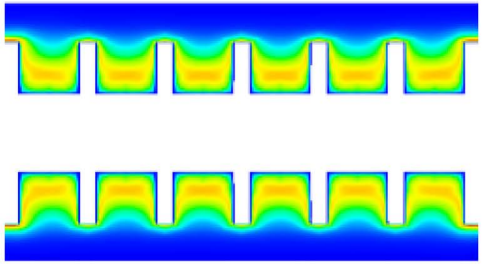

(d)

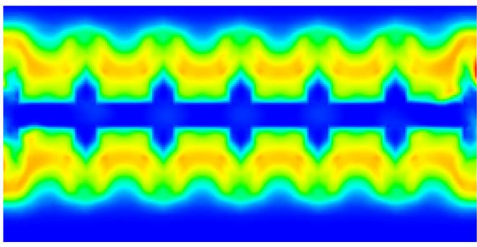

(e)

Figure 11. Instantaneous TKE distribution. (a) C1.2 at $x / c=0.9$; (b) C1.2 at $x / c=1$; (c) $\mathrm{C} 2$ at $x / c=0.73$; (d) C2 at $x / c=0.9$; (e) C2 at $x / c=1$.

1.5 meters directly above the airfoils' TEs. Discrete FFT is performed on the resulting time signals seen to compute the Sound Pressure Level (SPL) signal in the frequency domain, as shown in Figure 12. The Hanning window is applied to the time signal to reduce numerical leakages associated with the discrete FFT [47]. Both cases exhibit broadband behavior. No major discrepancies are seen in terms of general trend and no tonal peaks are present. A band-pass filter was applied obtained signals for $\mathrm{C} 1.2$ and $\mathrm{C} 2$ to calculate the overall sound pressure level (OASPL) for different frequency ranges, as defined in Equation (23). An OASPL reduction of $0.488 \mathrm{~dB}$ was predicted in the frequency range $1000 \mathrm{~Hz}$ to $5000 \mathrm{~Hz}$.

As part of the current study, and in addition to the numerical predictions, wind tunnel testing has been performed to evaluate the noise of finlets at different angles of attack. The airfoil has a chord length $c$ of $300 \mathrm{~mm}$, and the width is similar to the width of the nozzle exit at $510 \mathrm{~mm}$. The airfoil AOA is set to 0,5 , $-5,10$ and -10 degrees and fixed to the nozzle exit by two side plates. The microphone was fixed and placed at about $1.4 \mathrm{~m}$ from the TE at a polar angle of $90^{\circ}$ for all tests. The free jet velocity was set to $24 \mathrm{~m} / \mathrm{s}$ and the flow parameters and 


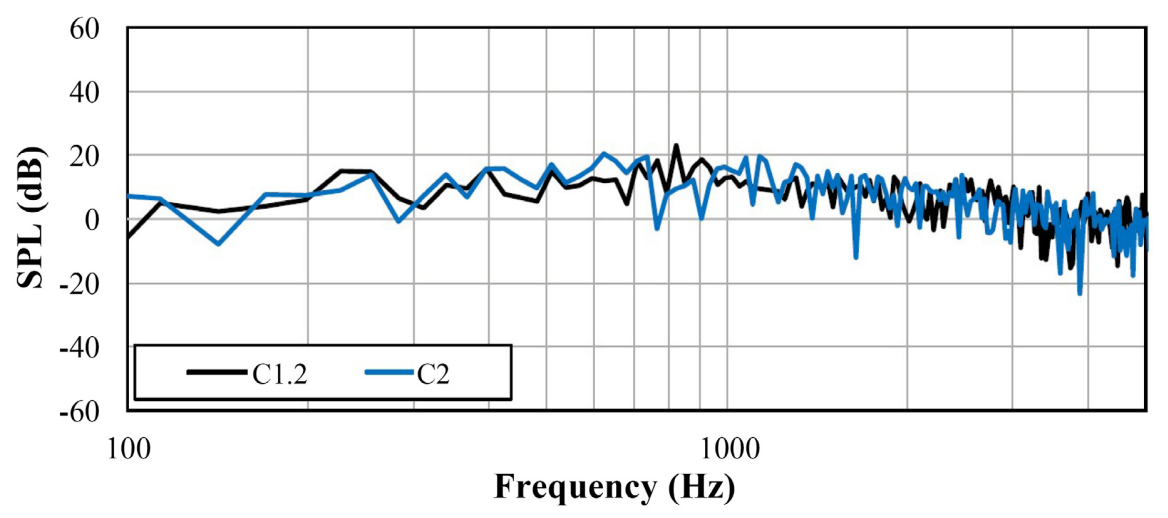

Figure 12. Simulated frequency domain noise signal.

chord length are similar to the computational cases, yielding a $\operatorname{Re}_{c}$ of approximately 500,000. The fluctuating pressure-time signals for the used microphone are recorded and then used to calculate the SPL spectrum. The data sampling frequency is set to $20 \mathrm{kHz}$ and the data sampling period is 30 seconds, corresponding to a frequency resolution of $0.033 \mathrm{~Hz}$. The obtained signal is also passed through a time-domain filter to remove the low and high frequency contamination, caused by the microphone's low frequency roll off and high-frequency aliasing. The band-pass filter used is a Butterworth filter with the first and second stopband frequencies of 100 and $\frac{f_{s}}{2} \mathrm{~Hz}$ respectively, where $f_{s}$ is the sampling frequency. The sound pressure level, SPL, is computed using the root mean square (RMS) of filtered pressure signal as following:

$$
\mathrm{SPL}=10 \log _{10}\left(\frac{P_{R M S}^{2}}{P_{\text {ref }}^{2}}\right)
$$

where: $P_{R M S}$ is the root mean square pressure, and $P_{r e f}$ is the standard reference pressure in air, $20 \mu \mathrm{Pa}$.

In Figure 13 the obtained SPL levels are displayed for AOA $=0^{\circ}, 5^{\circ},-5^{\circ}, 10^{\circ}$ and $-10^{\circ}$. For all the presented cases, the introduction of finlets did not alter the general shape of the acoustic spectra. The acoustic spectra kept the same broadband behavior, with slight differences in SPL amplitudes. The difference in amplitude between the two configurations is sensitive to the AOA, and is most pronounced in $\mathrm{AOA}= \pm 10^{\circ}$. Finlets did not affect the broadband behavior of the radiated noise and no tonal peaks are observed. The OASPL is calculated to better quantify the effect of finlets on the radiated noise. Table 3 shows the obtained noise reduction as a function of the airfoil AOAs, where:

$$
\triangle \mathrm{OASPL}=\mathrm{OASPL}(\text { finned TE })-\mathrm{OASPL}_{-}(\text {straight TE})
$$

It is shown that noise reduction increases as the $\mathrm{AOA}$ is increased, and that values are consistent for the same absolute angles $\left(\triangle \mathrm{OASPL}_{-5^{\circ}} \approx \triangle \mathrm{OASPL}_{5^{\circ}}\right.$ and $\triangle \mathrm{OASPL}_{-10^{\circ}} \approx \Delta \mathrm{OASPL}_{10^{\circ}}$. Since noise reduction is a function of the AOA, and modifying the AOA changes the BL thickness, then the noise reduction efficiency of finlets is dependent on the BL thickness. The observed trend agrees with 
Table 3. Noise reduction as a function of the airfoil AOAs.

\begin{tabular}{cc}
\hline $\mathrm{AOA}\left({ }^{\circ}\right)$ & $\Delta$ OASPL $(\mathrm{dB})$ \\
\hline-10 & -0.761 \\
-5 & -0.681 \\
0 & -0.337 \\
5 & -0.696 \\
10 & -0.782 \\
\hline
\end{tabular}
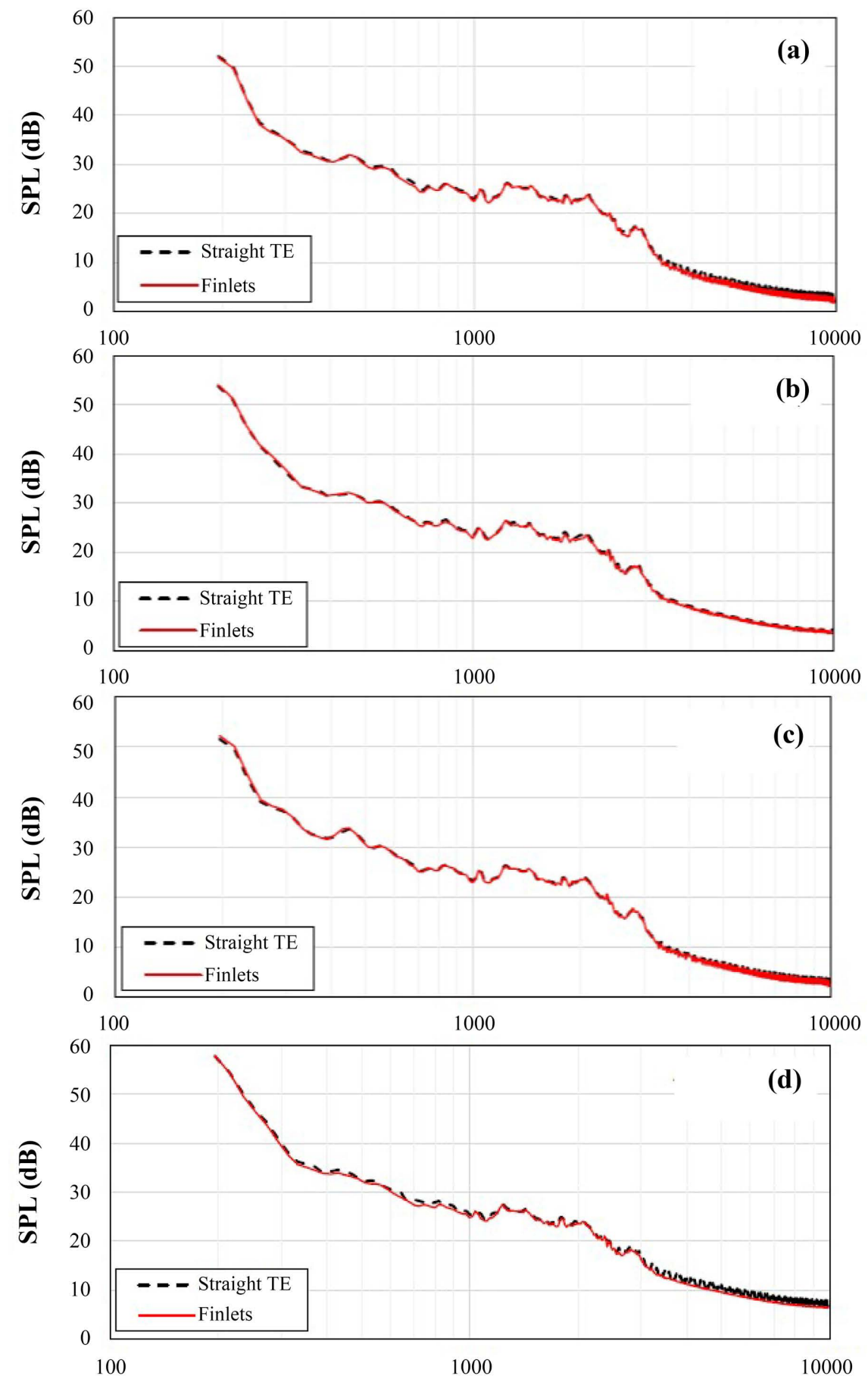


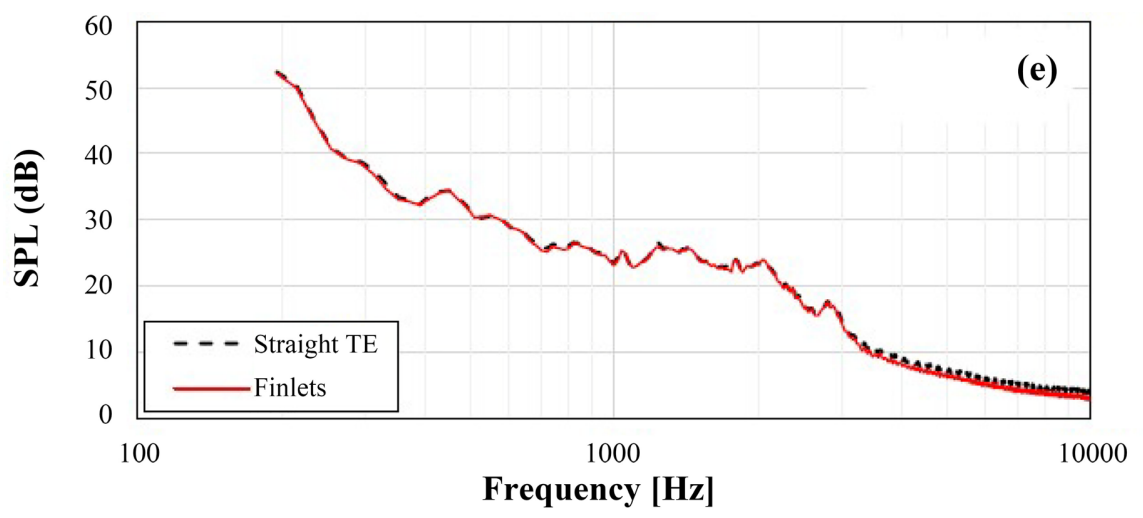

Figure 13. Experimentally obtained SPL levels for several AOAs: (a) $0^{\circ}$; (b) $5^{\circ}$; (c) $-5^{\circ}$; (d) $10^{\circ}$ and $(\mathrm{e})-10^{\circ}$.

the findings of Gstrein et al. [48]. Experimental results confirm that finlets reduce the generated broadband noise. Even tough the overall noise reduction is not significant (as shown in Table 3), it is important to note that this solution can potentially become attractive to systems that contain several or extensive trailing edge structures or components, e.g., an aircraft wing or lifting surface, and a wind turbine.

\section{Conclusions}

Embedded large eddy simulations and experimental wind tunnel testing are carried out on a NACA0012 airfoil having different TE configurations. TE finlets were investigated for a freestream flow velocity $u_{\infty}=24 \mathrm{~m} / \mathrm{s}, \mathrm{AOA}=0$ and $R e_{c}$ of approximately 500,000. A mesh convergence study is performed and the obtained pressure coefficient distribution is validated. The turbulent boundary layer was decorrelated in the spanwise direction and the size of the counter-rotating eddies near the TE was reduced. The lift force fluctuations history shows that C1.2 and $\mathrm{C} 2$ are subject to random, broadband lift variations, contrary to what one observes in typical TE serrations, which usually exhibit sinusoidal fluctuations, hence causing tonal noise. In terms of the general trend, no tonal peaks were observed. An OASPL reduction of $0.488 \mathrm{~dB}$ was predicted in the frequency range from $1000 \mathrm{~Hz}$ to $5000 \mathrm{~Hz}$.

Wind tunnel tests showed the effect of the airfoil AOA on obtained sound reduction. For $\mathrm{AOA}=-10^{\circ},-5^{\circ}, 0^{\circ}, 5^{\circ}$ and $10^{\circ}$, OASPL reductions of $0.761 \mathrm{~dB}$, $0.681 \mathrm{~dB}, 0.337 \mathrm{~dB}, 0.696 \mathrm{~dB}$ and $0.782 \mathrm{~dB}$ were obtained, respectively. $\triangle \mathrm{OASPL}$ between a flat TE and a finned TE was shown to be sensitive to the AOA, and is the largest at $\mathrm{AOA}= \pm 10^{\circ}$. The experimental results confirmed the dependence of finlet behavior on the thickness of the BL. The reductions were also consistent for the same absolute AOAs. Qualitative agreement between numerical and experimental results was achieved, in terms of spectral characteristics and broadband behavior. The effect of finlets deformed and decorrelated the large coherent structures of the TBL before they reached the airfoil TE, decreasing the velocity of the fluid particles around the sharp TE, thus reducing the efficiency of acoustic scat- 
tering and provoking a broadband noise reduction.

\section{Conflicts of Interest}

The authors declare no conflicts of interest regarding the publication of this paper.

\section{References}

[1] Ortega, C.P. (2012) Chapter 2: Effects of Noise Pollution on Birds: A Brief Review of Our Knowledge. In: Ornithological Monographs, Vol. 74, American Ornithological Society, USA, 6-22. https://doi.org/10.1525/om.2012.74.1.6

[2] Bronzaft, A.L. (2004) Noise Pollution: A Threat to Our Mental and Physical Well-Being. The Journal of the Acoustical Society of America, 115, 2567-2567. https://doi.org/10.1121/1.4784049

[3] Jianu, O., Naterer, G. and Rosen, M. (2011) Noise Pollution Prevention in Wind Turbines: Status and Recent Advances. Proceedings of the 1 st World Sustainability Forum (on line), 1-30 November.

[4] Graham, R.R. (1934) The Silent Flight of Owls. The Journal of the Royal Aeronautical Society, 38, 837-843. https://doi.org/10.1017/S0368393100109915

[5] Lilley, G. (1998) A Study of the Silent Flight of the Owl. 4th AIAA/CEAS Aeroacoustics Conference, Toulouse, 2-4 June 1998, AIAA 98-2340. https://doi.org/10.2514/6.1998-2340

[6] Clark, I.A., Alexander, W.N., Devenport, W., Glegg, S., Jaworski, J.W., Daly, C. and Peake, N. (2017) Bioinspired Trailing-Edge Noise Control. AIAA Journal, 55, 740-754. https://doi.org/10.2514/1.J055243

[7] Afshari, A., Azarpeyvand, M., Dehghan, A.A. and Szoke, M. (2017) Effects of Streamwise Surface Treatments on Trailing Edge Noise Reduction. 23rd AIAA/CEAS Aeroacoustics Conference, Denver, 5-9 June 2017, AIAA 2017-3499. https://doi.org/10.2514/6.2017-3499

[8] Manoha, E., Troff, B. and Sagaut, P. (2000) Trailing-Edge Noise Prediction Using Large-Eddy Simulation and Acoustic Analogy. AIAA Journal, 38, 575-583. https://doi.org/10.2514/2.1015

[9] Agrawal, B.R. and Sharma, A. (2016) Numerical Investigations of Bio-Inspired Blade Designs to Reduce Broadband Noise in Aircraft Engines and Wind Turbines. 54th AIAA Aerospace Sciences Meeting, San Diego, 4-8 January 2016, AIAA 2016-0760. https://doi.org/10.2514/6.2016-0760

[10] Tang, H., Lei, Y. and Fu, Y. (2019) Noise Reduction Mechanisms of an Airfoil with Trailing Edge Serrations at Low Mach Number. Applied Sciences, 9, 3784. https://doi.org/10.3390/app9183784

[11] Zilstra, A. and Johnson, D. (2019) LES and FW-H Prediction of Aeroacoustic Noise for a SD 7037 Airfoil for Wind Turbine Applications. 25th AIAA/CEAS Aeroacoustics Conference, Delft, 20-23 May 2019, AIAA 2019-2537. https://doi.org/10.2514/6.2019-2537

[12] Bodling, A. and Sharma, A. (2018) Numerical Investigation of Low-Noise Airfoils Inspired by the Down Coat of Owls. Bioinspiration \& Biomimetics, 14, Article ID: 016013. https://doi.org/10.2514/6.2018-3925

[13] Rumpfkeil, M.P. (2017) Using Steady Flow Analysis for Noise Predictions. Computers \& Fluids, 154, 347-357. https://doi.org/10.1016/j.compfluid.2017.03.003 
[14] Quéméré, P. and Sagaut, P. (2002) Zonal Multi-Domain RANS/LES Simulations of Turbulent Flows. International Journal for Numerical Methods in Fluids, 40, 903-925. https://doi.org/10.1002/fld.381

[15] Terracol, M. (2006) A Zonal RANS/LES Approach for Noise Sources Prediction. Flow, Turbulence and Combustion, 77, 161-184.

https://doi.org/10.1007/s10494-006-9042-6

[16] Fröhlich, J. and von Terzi, D. (2008) Hybrid LES/RANS Methods for the Simulation of Turbulent Flows. Progress in Aerospace Sciences, 44, 349-377. https://doi.org/10.1016/j.paerosci.2008.05.001

[17] Kim, T., Jeon, M., Lee, S. and Shin, H. (2014) Numerical Simulation of Flatback Airfoil Aerodynamic Noise. Renewable Energy, 65, 192-201.

https://doi.org/10.1016/j.renene.2013.08.036

[18] Lane, G., Croaker, P. and Ding, Y. (2019) Embedded Large Eddy Simulation Method for Predicting Flow-Induced Noise. Proceedings of ACOUSTICS, Cape Schanck, 10-13 November 2019, 1-10.

[19] Zuo, Z.G., Huang, Q. and Liu, S. (2019) An Analysis on the Flow Field Structures and the Aerodynamic Noise of Airfoils with Serrated Trailing Edges Based on Embedded Large Eddy Flow Simulations. Journal of Applied Fluid Mechanics, 12, 327-339. https://doi.org/10.29252/jafm.12.02.29142

[20] ANSYS (2019) Fluent 2019 Theory Guide. ANSYS, Inc., Canonsburg.

[21] Leonard, A. (1975) Energy Cascade in Large-Eddy Simulations of Turbulent Fluid Flows. Advances in Geophysics, 18, 237-248. https://doi.org/10.1016/S0065-2687(08)60464-1

[22] Hinze, J.O. (1987) Turbulence. McGraw-Hill, New York.

[23] Nicoud, F. and Ducros, F. (1999) Subgrid-Scale Stress Modelling Based on the Square of the Velocity Gradient Tensor. Flow, Turbulence and Combustion, 62, 183-200. https://doi.org/10.1023/A:1009995426001

[24] Hughes, R.L. (2007) A Mathematical Determination of von Karman's Constant, K. Journal of Hydraulic Research, 45, 563-566. https://doi.org/10.1080/00221686.2007.9521792

[25] Bailey, S.C.C., et al. (2014) Estimating the Value of von Kármán's Constant in Turbulent Pipe Flow. Journal of Fluid Mechanics, 749, 79-98. https://doi.org/10.1017/jfm.2014.208

[26] Liu, Z., Wu, Y. and Li, B. (2020) An Assessment on the Performance of Sub-Grid Scale Models of Large Eddy Simulation in Modeling Bubbly Flows. Powder Technology, 374, 470-481. https://doi.org/10.1016/j.powtec.2020.07.055

[27] Mathey, F., Cokljat, D., Bertoglio, J.P. and Sergent, E. (2006) Assessment of the Vortex Method for Large Eddy Simulation Inlet Conditions. Progress in Computational Fluid Dynamics, 6, 58-67. https://doi.org/10.1504/PCFD.2006.009483

[28] Lighthill, M.J. (1952) On Sound Generated Aerodynamically. Proceedings of the Royal Society of London. Series A, Mathematical and Physical Sciences, 211, 564-587. https://doi.org/10.1098/rspa.1952.0060

[29] Ffowcs-Williams, J.E. and Hawkings, D.L. (1969) Sound Generation by Turbulence and Surfaces in Arbitrary Motion. Proceedings of the Royal Society of London. Series A, Mathematical and Physical Sciences, 264, 321-342. https://doi.org/10.1098/rsta.1969.0031

[30] Brentner, K.S. and Farassat, F. (1998) Analytical Comparison of the Acoustic Analogy and Kirchhoff Formulation for Moving Surfaces. AIAA Journal, 36, 1379-1386. 
https://doi.org/10.2514/2.558

[31] Shi, Y.J. and Lee, S. (2020) Numerical Study of 3-D Finlets Using Reynolds-Averaged Navier-Stokes Computational Fluid Dynamics for Trailing Edge Noise Reduction. International Journal of Aeroacoustics, 19, 95-118. https://doi.org/10.1177/1475472X20905053

[32] Berger, M. and Aftosmis, M. (2012) Progress towards a Cartesian Cut-Cell Method for Viscous Compressible Flow. 50th AIAA Aerospace Sciences Meeting Including the New Horizons Forum and Aerospace Exposition, Nashville, 9-12 January 2012, AIAA 2012-1301. https://doi.org/10.2514/6.2012-1301

[33] Ghmati, R.E., Ali Jawad, B. and Koutsavdis, E. (2012) An Investigation of CutCell Meshing Strategies for Accurate Aerodynamic Performance Prediction. SAE International Journal of Passenger Cars-Mechanical Systems, 5, 369-380. https://doi.org/10.4271/2012-01-0499

[34] Menter, F.R. (2012) Best Practice: Scale-Resolving Simulations in ANSYS CFD. ANSYS Germany GmbH, Darmstadt, 1.

[35] ANSYS (2019) Fluent 2019 User Guide. ANSYS, Inc., Canonsburg.

[36] Menter, F.R. (1994) Two-Equation Eddy-Viscosity Turbulence Models for Engineering Applications. AIAA Journal, 32, 1598-1605. https://doi.org/10.2514/3.12149

[37] Menter, F.R. (2015) Best Practice: Scale-Resolving Simulations in ANSYS CFD. ANSYS Germany GmbH, Darmstadt.

[38] Al Tlua, B. and Rocha, J. (2019) Development and Testing of an Aeroacoustic Wind Tunnel Test Section. Canadian Acoustics, 47, 64-65.

[39] Al Tlua, B. and Rocha, J. (2020) Optimization and Testing of Flat-Plate Trailing-Edge Serration Geometry for Reducing Airfoil Self-Noise. Canadian Acoustics, 48, 7-17.

[40] Clauser, F.H. (1954) Turbulent Boundary Layers in Adverse Pressure Gradients. Journal of the Aeronautical Sciences, 21, 91-108. https://doi.org/10.2514/8.2938

[41] Falkner, V.M. and Skan, W. (1930) Some Approximate Solutions of the Boundary Layer Equations. A.R.C Reports and Memoranda, No. 1314.

[42] Lee, H. and Kang, S.H. (2000) Flow Characteristics of Transitional Boundary Layers on an Airfoil in Wakes. Journal of Fluids Engineering, 122, 522-532.

https://doi.org/10.1115/1.1287592

[43] Marsden, O., Bogey, C. and Bailly, C. (2006) Direct Noise Computation around a 3-D NACA 0012 Airfoil. 12th AIAA/CEAS Aeroacoustics Conference (27th AIAA Aeroacoustics Conference), Cambridge, 8-10 May 2006, AIAA 2006-2503.

https://doi.org/10.2514/6.2006-2503

[44] Chong, M.S., Perry, A.E. and Cantwell, B.J. (1990) A General Classification of ThreeDimensional Flow Fields. Physics of Fluids A: Fluid Dynamics, 2, 765-777. https://doi.org/10.1063/1.857730

[45] Amiet, R.K. (1976) Noise Due to Turbulent Flow past a Trailing Edge. Journal of Sound and Vibration, 47, 387-393. https://doi.org/10.1016/0022-460X(76)90948-2

[46] Williams, J.E.F. and Hall, L.H. (1970) Aerodynamic Sound Generation by Turbulent Flow in the Vicinity of a Scattering Half Plane. Journal of Fluid Mechanics, 40, 657-670. https://doi.org/10.1017/S0022112070000368

[47] Press, W.H., Flannery, B.P., Teukolsky, S.A. and Vetterling, W.T. (1986) Numerical Recipes. Cambridge University Press, Cambridge.

[48] Gstrein, F., Zang, N. and Azarpeyvand, M. (2020) Application of Finlets for Trailing Edge Noise Reduction of a NACA 0012 Airfoil. AIAA A viation 2020 Forum (virtual event), 15-19 June 2020, AIAA 2020-2502. https://doi.org/10.2514/6.2020-2502 


\section{Nomenclature}

AOA: Angle of attack;

BL: Boundary layer;

CFD: Computational fluid dynamics;

c. Airfoil chord length;

$C_{p}$ : Pressure coefficient;

$s$. Finlet spacing;

$d t$. Time step;

t. Time;

$f_{s}$ : Sampling frequency;

f. frequency;

$u, v, w$. Fluid velocity;

$P$. Fluid pressure;

$M_{\infty}:$ Mach number;

$\operatorname{Re}_{c}$ : Chord based Reynolds number;

$\mu$ : Dynamic viscosity;

v. Kinematic viscosity;

$L$ : Length;

$Q$ : Second invariant of velocity gradient tensor;

$\rho$ : Density;

SGS: Subgrid-scale;

TE: Trailing-edge;

$T_{\mathrm{FT}}$ : Through-flow time;

$\omega$ : Vorticity. 